\title{
Two-Dimensional Simulation of Electrospun Nanofibrous Structures: Connection of Experimental and Simulated Results
}

\author{
Panu Danwanichakul and Duangkamol Danwanichakul \\ Department of Chemical Engineering, Faculty of Engineering, Thammasat University, 99 Moo 18, Phaholyothin Road, \\ Klong Nueng, Klong Luang, Pathum Thani 12120, Thailand \\ Correspondence should be addressed to Panu Danwanichakul; dpanu@engr.tu.ac.th
}

Received 23 May 2013; Revised 22 October 2013; Accepted 29 October 2013; Published 20 January 2014

Academic Editor: Davide Vione

Copyright (C) 2014 P. Danwanichakul and D. Danwanichakul. This is an open access article distributed under the Creative Commons Attribution License, which permits unrestricted use, distribution, and reproduction in any medium, provided the original work is properly cited.

\begin{abstract}
Nanofibrous mats were obtained from electrospinning Nylon-6 solutions with concentrations of 30 and 35 wt $\%$ and were tested for filtration of polystyrene particles in suspension. Some experimental results were compared with the simulated ones. In the simulation, the two-dimensional structures were constructed by randomly depositing a nanofiber, which was assumed as an ellipse with an aspect ratio of 100 , one by one. The nanofiber size is assumed to be polydisperse. The results showed that simulated configurations resembled real nanofibers with polydisperse diameters. Fibers from higher solution concentration were larger, resulting in larger pore size, which was confirmed with simulations. Varying the size distribution around the same average value did not make any difference to the surface coverage but it affected 2D pore areas for the systems at low fiber density. In addition, the probability for a particle to pass through the porous structure was less when the fiber density was higher and the particle diameter was larger, which was consistent with the filtration test. Lastly, water flux measurement could yield the void volume fraction as well as the volume-averaged pore diameter, which was found to be greater than the averaged 2D pore diameter from SEM micrographs by the quantity related to the fiber size.
\end{abstract}

\section{Introduction}

Electrospinning technique is an easy method to produce nanofibers with size in submicron or nanometer range, by applying the electrical force generated by the electric field between the end of the metal needle and the ground collector. The liquid source for spinning fibers may be a polymer melt or a polymer solution. A polymer melt or a polymer solution is placed in a syringe. Once the applied electrical force is greater than the surface tension of the hanging hemisphere drop at the end of the syringe needle, the liquid is pulled out of the needle and swirled onto the collector surface. Continuous fibers are produced in this manner. While the solvent evaporates, the final nonwoven nanofibrous structure is solidified. A large number of papers discussing the production of nanofibers from various polymer systems and their applications have been published [1-5]. The research on nanofibers is still receiving more and more attention. Indeed, the benefit of large surface area to volume ratio of nanofibrous structures improves their performance in many aspects including higher mechanical strength, an increase in surface activity, and an increase in adsorption capacity. Therefore, nanofibers were widely studied for use as sensors [1], catalysts [2], drug delivery systems [3], tissue scaffolds [4], and filters [5]. The study about surface characterization and pore generation of nanofibrous membranes is, thus, of great interest.

There are many factors influencing the size of fibers produced via electrospinning. Those are voltage of power supply, distance between the needle tip and the ground collector, solution flow rate, viscosity, conductivity, and surface tension of polymer solutions. Some of these process parameters were modeled by Danwanichakul et al. by using poly(vinyl alcohol) as a model polymer [6]. However, not only fibers but also the pores generated by the overlap of fibers themselves are of importance. H.-S. Park and Y. O. Park found that pore size distribution in nanofibrous structures was narrow or sometimes monodisperse [7]. The experiment was performed but there was no explanation for that. The theoretical work 
could then fill the gap. Eichhhorn and Sampson simulated the fibers by assuming that they are of cylindrical shape whose diameter is much smaller than the length [8]. They found that if the mass of fibers per unit area was fixed, when the mass of the fibers increased, the average pore size increased. Maze et al. simulated the filter using nanofibers with a diameter of $200 \mathrm{~nm}$ and allowed the particles with diameters between 50 and $500 \mathrm{~nm}$ to pass through the pores. They found that the pressure drop was related to the pore size as could be predicted by fluid mechanics [9]. In addition to 2D simulations, Hosseini and Tafreshi attempted to simulate 3D nanofibrous structures by building rigid cylindrical fibers one by one on top of each other and used computational fluid dynamics to study the collection of particles in the air when passing through these simulated filters [10]. Another attempt to create 3D nanofibrous structure was made by Sambaer et al. in the simulation of filtration process via polyurethane nanofibers [11]. To construct fiber mats, an image of top layer of fibers was used as a template for creating the whole mats with multiple layers. This method was proved successful in imitating the real behavior of the nanofibers in filtration process.

The success of 3D-simulation by Sambaer et al. [11] was, however, based on the 2D structures of the nanofiber network so the knowledge of $2 \mathrm{D}$ structures is still important. All papers about simulation of electrospun nanofibers involved the filtration of aerosol and assumed that the fiber size was monodisperse. In this work, nylon- 6 was selected as a model polymer and smooth nanofibers produced by electrospinning were applied in filtration of particles from liquid suspension. The 2D simulation was performed to study the effect of fiber size polydispersity on the pore size of the nanostructures as well as to connect the simulated results with the experimental results either qualitatively or quantitatively.

\section{Material and Method}

2.1. Materials. Nylon-6 was purchased from Sigma-Aldrich Co. LLC. Formic acid was supplied by Ajax Finechem Pty Ltd. The suspensions of polystyrene particles with average particle sizes of $90 \mathrm{~nm}(50-100 \mathrm{~nm})$ and $200 \mathrm{~nm}(200-300 \mathrm{~nm})$ were purchased from Spherotech, Inc. All chemicals were of analytical grade and used without further purification.

2.2. Electrospinning of Nylon-6 Nanofibers. Nylon-6 was dissolved in $10 \mathrm{~mL}$ of $90 \mathrm{wt} \%$ formic acid to obtain the spinning solutions with concentrations of 30 and $35 \mathrm{wt} \% / \mathrm{vol}$, namely, N6-30 and N6-35, respectively. Each mixture was blended with a magnetic stirrer at room temperature for $2-4 \mathrm{~h}$ until the solution was homogeneous. The spinning solution was poured into a $2 \mathrm{~mL}$ syringe with a needle whose inner diameter and length were $0.514 \mathrm{~mm}$ and $4.2 \mathrm{~cm}$, respectively. A high voltage power supply (Glassman PS/MJ30PO400) was connected to the needle end by a stainless steel electrode. Another electrode was connected to the stationary collector covered with $3.5 \times 3.5 \mathrm{~cm}^{2}$ copper plate. The voltage was set at $21 \mathrm{kV}$ and the distance between the needle tip and the collector was held constant at $9 \mathrm{~cm}$. The spinning time was about $1.5-3 \mathrm{~h}$ in order to obtain the $0.1 \pm 0.02 \mathrm{~mm}$ thick nanofiber mats. The spinning was performed without a syringe pump so the polymer solution was purely pulled out of the needle tip by electrical forces. However, the flow rates could be measured to be 3.33 and $1.94 \mu \mathrm{L} / \mathrm{min}$ for N6-30 and N6-35 solutions, respectively, reflecting the effect of solution viscosity.

2.3. Morphology of Nanofibers. The morphology of nanofibers was observed with scanning electron microscope (SEM) (JEOL JSM-6310F) after coating by a gold sputtering coater for 100 seconds. Each SEM figure was analyzed for the average fiber size as well as the apparent average pore size of the structure. The fiber diameter and pore size were measured by comparing with the scale bar on the SEM figures. The average was from at least 100 samples. The fiber diameter was measured along the axis perpendicular to the fiber length and the pore size was measured along the horizontal axis by adopting the widest distance as the pore size. The $2 \mathrm{D}$ pore area could be determined rigorously by filling up the pore with small circular discs whose areas were known. The number of the discs reflects the area of the pore. To obtain the fiber coverage on the surface, the grey scale analysis was performed with the simulated figures. Fibers and vacant areas can be differentiated by the color so the fraction of deposited area could be determined.

2.4. Water Flux Measurement. In this experiment, a syringe containing $5 \mathrm{~mL}$ water was vertically connected to a filter holder inside which the filter medium was placed. A $0.5 \mathrm{~kg}$ piece of metal was then placed at the end of the syringe, pushing the water to pass through the circular filter medium whose diameter is $13 \mathrm{~mm}$. The flow is so slow that it could be considered a laminar flow and the volumetric flow rate of water could be measured. Pores inside the nanofibrous structure are usually interconnected so the structure resembles a bundle of tortuous tubes. The volume-averaged pore size could be determined using the relationship between water flux and pressure drop across the membrane as the expression in the following:

$$
\frac{d^{2}}{32}=\frac{U_{o} \mu \tau z}{\varepsilon \Delta P} .
$$

In (1), $U_{o}$ is the superficial velocity $(\mathrm{m} / \mathrm{s}), \mu$ is the viscosity of water at $30^{\circ} \mathrm{C}(\mathrm{Pa} \cdot \mathrm{s}), z$ is the filter thickness $(\mathrm{m}), \Delta P$ is pressure drop $(\mathrm{Pa}), \varepsilon$ is the void fraction, $\tau$ is the tortuosity, and $d$ is the volume-averaged pore diameter $(\mathrm{m})$.

2.5. Filtration of Polystyrene Particles. Firstly, the nanofiber filter was placed in the filter holder with a diameter of $13 \mathrm{~mm}$ and $5 \mathrm{~mL}$ of $200 \mathrm{ppm}$ polystyrene particle suspension was pumped through the filter by a syringe pump at a fixed flow rate of $8 \mu \mathrm{L} / \mathrm{sec}$. The polystyrene (PS) colloidal particles were collected by the filter and the concentration of the filtrate was measured by using the calibration curve showing the linear relationship between the concentration and absorbance measured with UV-VIS spectrophotometer at $490 \mathrm{~nm}$. The filtration efficiency is expressed as

$$
\text { Filtration efficiency }(\%)=1-\frac{C_{\text {filtrate }}}{C_{\text {initial }}} \times 100 \text {. }
$$


Here, $C_{\text {initial }}$ and $C_{\text {filtrate }}$ are the initial concentration of PS particles in suspension and the concentration in the filtrate coming out of the filter, respectively.

2.6. Computer Simulation of Nanofibrous Structures. The computer program was written in FORTRAN in this study. The deposition of fibers on a surface was performed by Monte Carlo simulations. First, the position on the 2D system and the size of fibers are randomly sampled one by one. Each "2D" fiber is modeled as an ellipse whose aspect ratio, that is, the ratio of the long axis length to the short axis length, equals to 100 . The length of short axis of the reference fiber is set at $d_{f}$ which is scaled with a unit size $d$ as $0.075 d$. The deposited area is scaled as $100 d^{2}$, equivalent to the area of the surface whose width and length are equally $10 \mathrm{~d}$. Therefore, the fiber size is $150 \mathrm{~nm}$ and the deposited square area is $5 \times 5 \mu \mathrm{m}^{2}$ when the size $d$ is set as $500 \mathrm{~nm}$. Not only the position of the fiber center but also the angle of the fiber major axis with respect to the $x$-axis is sampled randomly on the surface.

To study the effect of fiber size, the size polydispersity of the fibers was varied according to the uniform size distribution to be $0.85 d_{f}-1.15 d_{f}, 0.75 d_{f}-1.25 d_{f}, 0.65 d_{f}-1.35 d_{f}$, and $0.55 d_{f}-1.45 d_{f}$, where $d_{f}$ is the size of reference fiber which is $0.075 d$ as described above. The simulated structures were analyzed for the surface coverage on the deposited area and the average pore size of the structures when more fibers were deposited.

To quantify how difficult the particles will pass through the $2 \mathrm{D}$ porous structures as in the filtration test, the number of attempts for the particle to deposit on the structure was measured until it could successfully pass the $2 \mathrm{D}$ pore. The number of attempts reflects the probability of capturing the particles inside the nanostructures. In this test, the particle diameter was varied as $d, 0.75 d$, and $0.40 d$ and the fiber diameter was fixed at $d_{f}$.

\section{Results and Discussion}

3.1. Morphology of Nanofibers. SEM micrographs of electrospun products from solutions of different nylon- 6 concentrations are shown in Figure 1. The left-handed side represents the structures from $30 \mathrm{wt} \%$ nylon solution while the righthanded side was from $35 \mathrm{wt} \%$ solution. The image analysis of SEM figures yielded the average diameters of fibers and pores of the structures, which are also shown in the figure. Upon increasing the concentration from $30 \mathrm{wt} \%$ to $35 \mathrm{wt} \%$, the average diameter was increased from 103 to $144 \mathrm{~nm}$ because of an increase in solution viscosity [12]. It is clearly seen from the SEM figure and the size distributions of both fibers and pores that the larger fibers could form the structures with larger pores [13]. The average pore size was estimated to be $94 \mathrm{~nm}$ and $154 \mathrm{~nm}$ for N6-30 and N6-35, respectively.

3.2. The Simulated 2D Nanostructures. The number of deposited fibers on a unit area is defined as the number density of fibers. For instance, the system with number density of 0.1 contains 10 fibers on $100 d^{2}$ area. The structures are shown in Figure 2 where the number density increases from 0.1 to 0.8 for the structures composed of the same fiber size of $d_{f}$. However, since the actual structure contains fibers with polydisperse size as shown in Figure 1, the simulation was then done to obtain structures of various size distributions as displayed in Figure 3.

In Figure 3, all samples are compared at the same fiber number density of 0.8 . It could be seen that the fiber size could be differentiated from one another more easily as the size distribution is wider and this realization is similar to what is seen in the SEM figures. The information that could be obtained from the simulated figures using the gray scale analysis is the surface coverage of the nanofibers at different number densities. The knowledge of surface coverage is important in the applications of surface coating with nanofibers in order to modify the hydrophilicity or hydrophobicity of the original surfaces by using more hydrophilic or more hydrophobic nanofibers. For instance, the superhydrophobicity of the surface confirmed with water contact angle measurement was reported when coating fluorine-plasma-treated cellulose nanofibers on microfiber, which possessed large surface areas [14]. Another example dealing with surface coverage is that the minimum amount of nanofibers was also deposited on top of the large fiber filter to increase the filtration efficiency [15].

3.3. The Closeness of Simulated Structures and the Real Nanofibers. In order to compare the 2D nanofibrous structures with the simulated ones, the system of N6-35 was chosen. The average fiber size is $144 \mathrm{~nm}$ while the simulated average fiber size, $d_{f}$, is $150 \mathrm{~nm}$ on $5 \times 5$ micron $^{2}$. As seen in Figure 1, the size distribution of the fibers ranges from $70 \mathrm{~nm}$ to $225 \mathrm{~nm}$ so the closest distribution for the simulated fibers chosen for comparison is $0.55 d_{f}-1.45 d_{f}$, which corresponds to $82.5 \mathrm{~nm}$ to $217.5 \mathrm{~nm}$. The simulated structure and its pore size distribution are displayed in Figure 4. It is seen that when the fiber number density is 0.3 , the pore size distributions of simulated and SEM figures (Figure 1) are consistent. The pore size from SEM figure could be as large as $670 \mathrm{~nm}$. The average pore size of simulated structure is $165 \mathrm{~nm}$ which is close to $154 \mathrm{~nm}$ of the SEM figures. Therefore, it is possible to simulate the electrospun nanofibers in $2 \mathrm{D}$ to make them resemble the real structure.

The comparison could also be made by fixing the solid volume fraction (SVF) or solidity of the structures with different fiber size distribution. The solid volume fraction ranges from very low to $13 \%$ [10]. The comparison of simulated structures is shown in Figure 5. The figure shows the structure of simulated nanofibers of the average size around $100 \mathrm{~nm}$, which corresponds to N6-30, and that with average size around $150 \mathrm{~nm}$, which corresponds to N6-35, at the same solid volume fraction. If the thickness of the membrane is assumed to be the same, the number density is then inversely proportional to the squared fiber diameter. Therefore, to be compared, the number densities of the two systems are 0.45 and 0.2 , respectively. It is evident that at the same solid volume fraction, the pore size of the structures containing large fibers is larger as previously seen in Figure 1. 

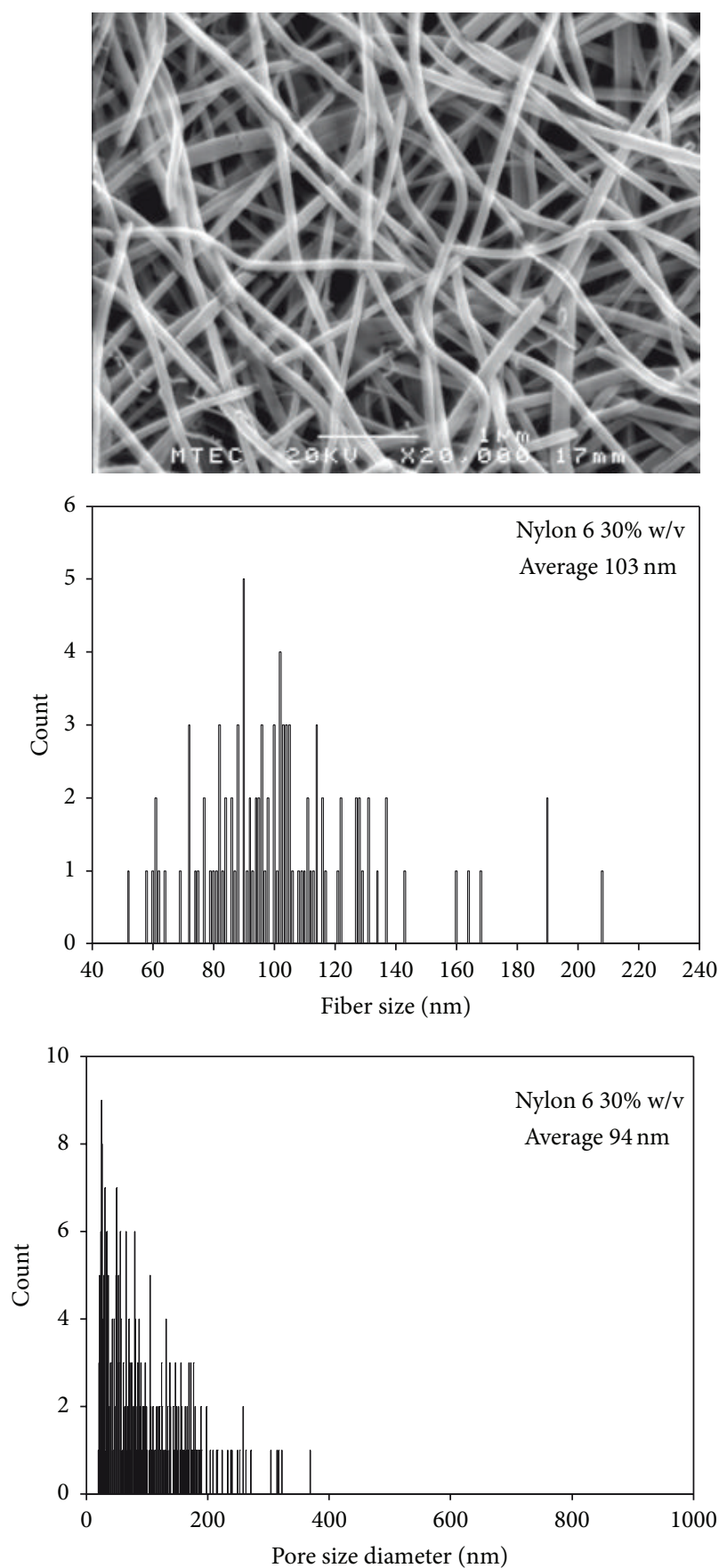

(a)
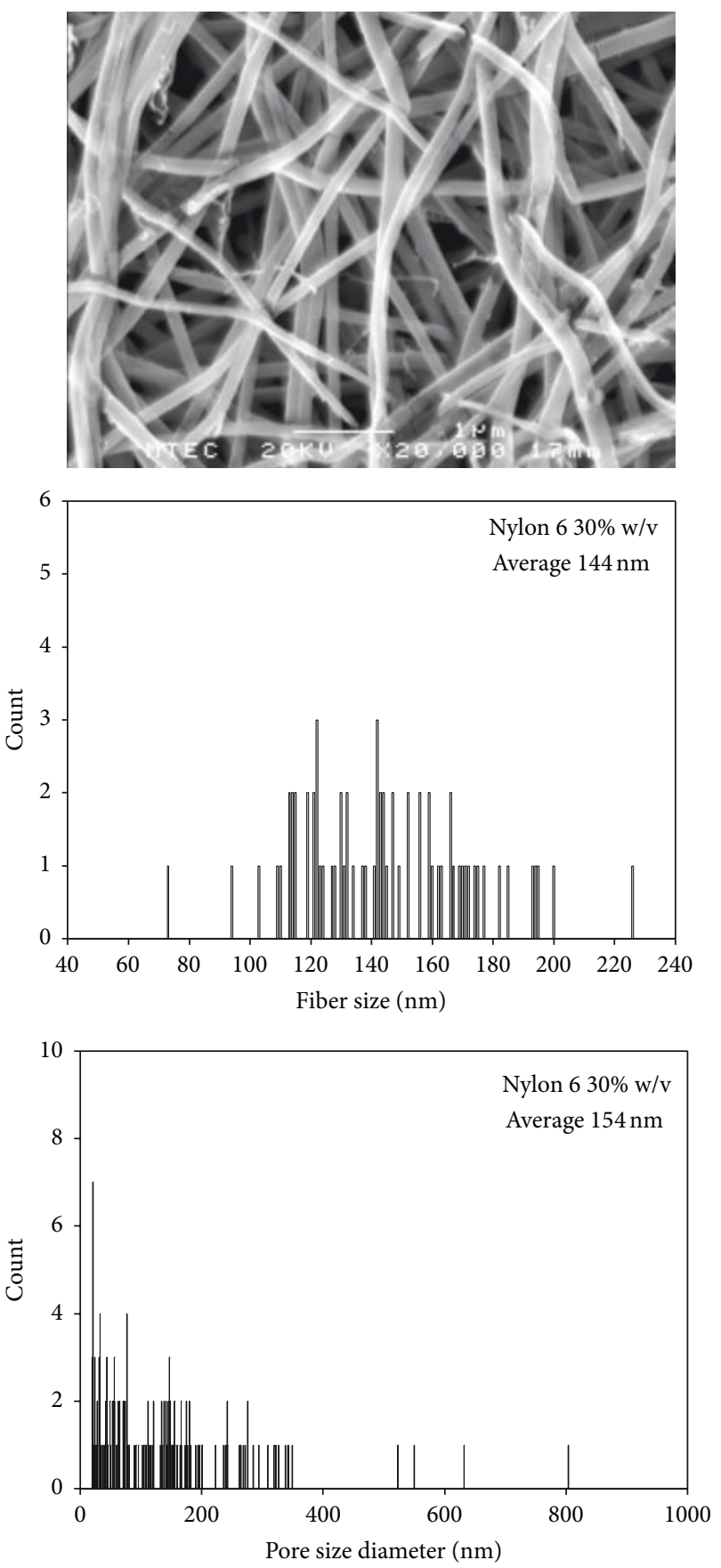

(b)

FIGURE 1: SEM figures of nylon-6 nanofibers electrospun from solutions of (a) $30 \mathrm{wt} \%$ and (b) $35 \mathrm{wt} \%$ along with the fiber and pore size distributions.

3.4. The Surface Coverage of Nanofibers and Projected Pore Area. The surface coverage and the average pore size of the corresponding simulated structures in Figure 3 are illustrated in Figures 6 and 7, respectively. The size of the fibers was varied according to a uniform distribution, where the lower limit and upper limit need to be specified. The sampled fiber size within this range will have the same probability. The chosen lower limits and upper limits of the fiber sizes are $0.85 d_{f}-1.15 d_{f}, 0.75 d_{f}-1.25 d_{f}, 0.65 d_{f}-1.35 d_{f}$, and
$0.55 d_{f}-1.45 d_{f}$. It should be noted that all uniform distributions applied in this work are around the same average fiber size of $d_{f}$, the value at the center of the two limits. Accordingly, the width of size distribution was wider as could be seen from the difference between the lower and upper limits which is $0.30 d_{f}, 0.50 d_{f}, 0.70 d_{f}$, and $0.90 d_{f}$, respectively.

The gray scale analysis with Photoshop was applied in this study. Differentiation between the deposited area (gray area) and the available area or porous area (white area) could be 

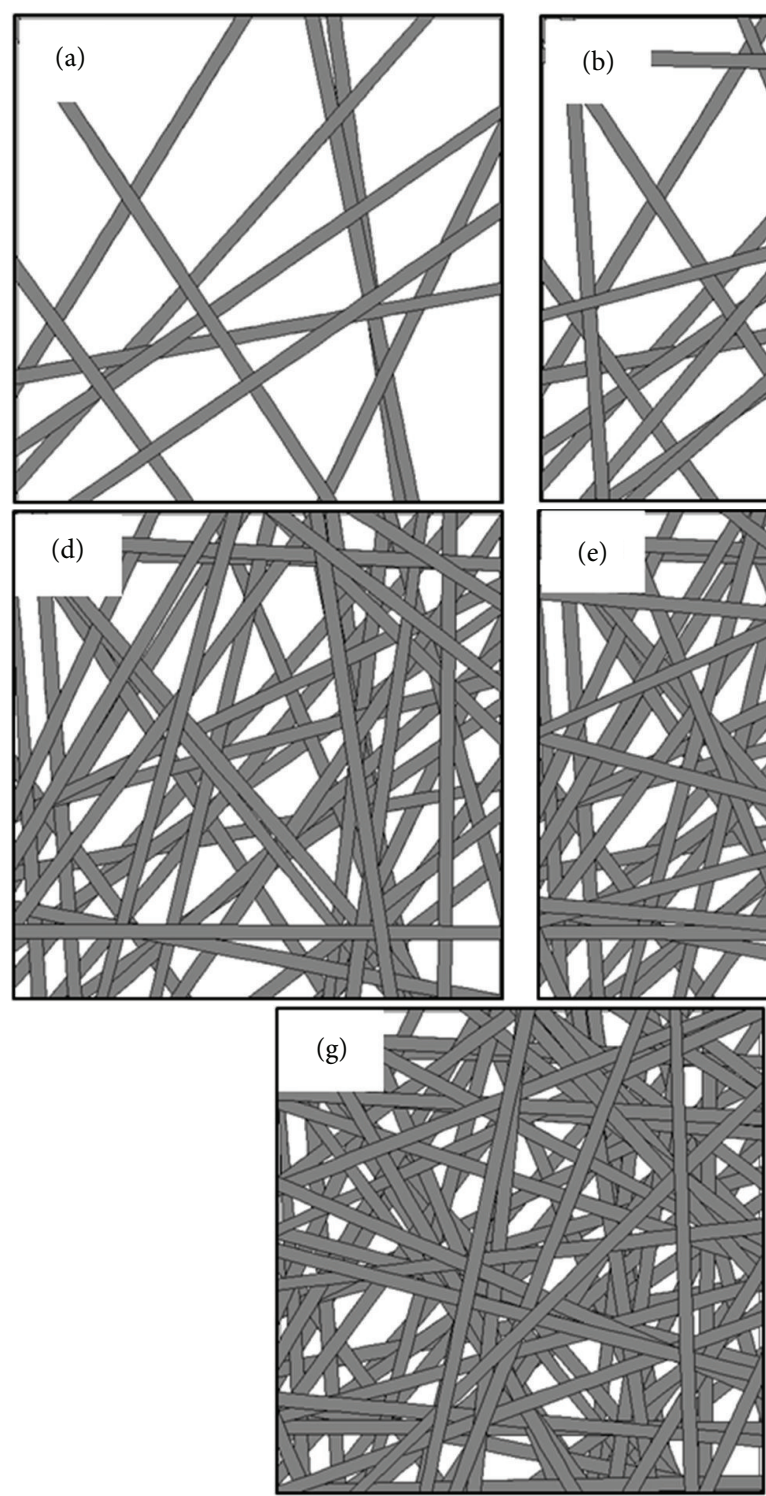
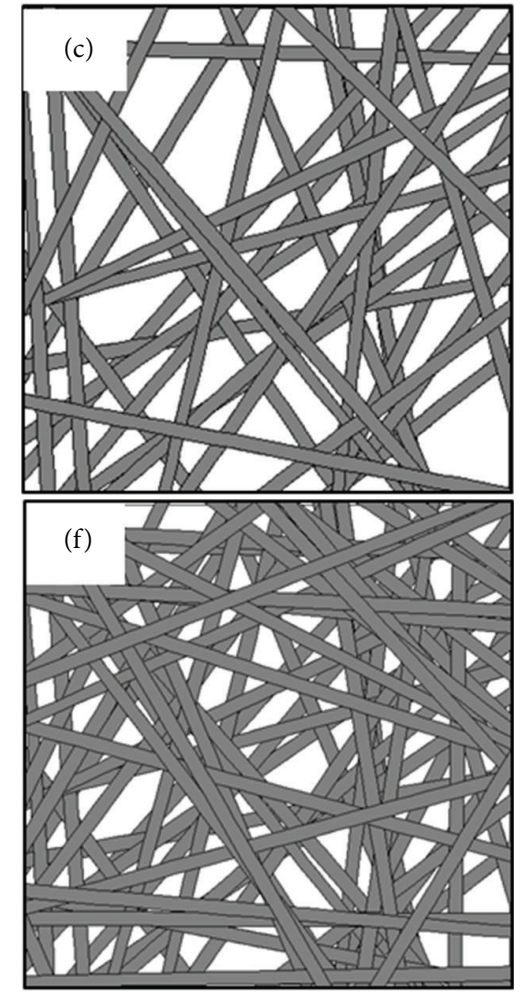
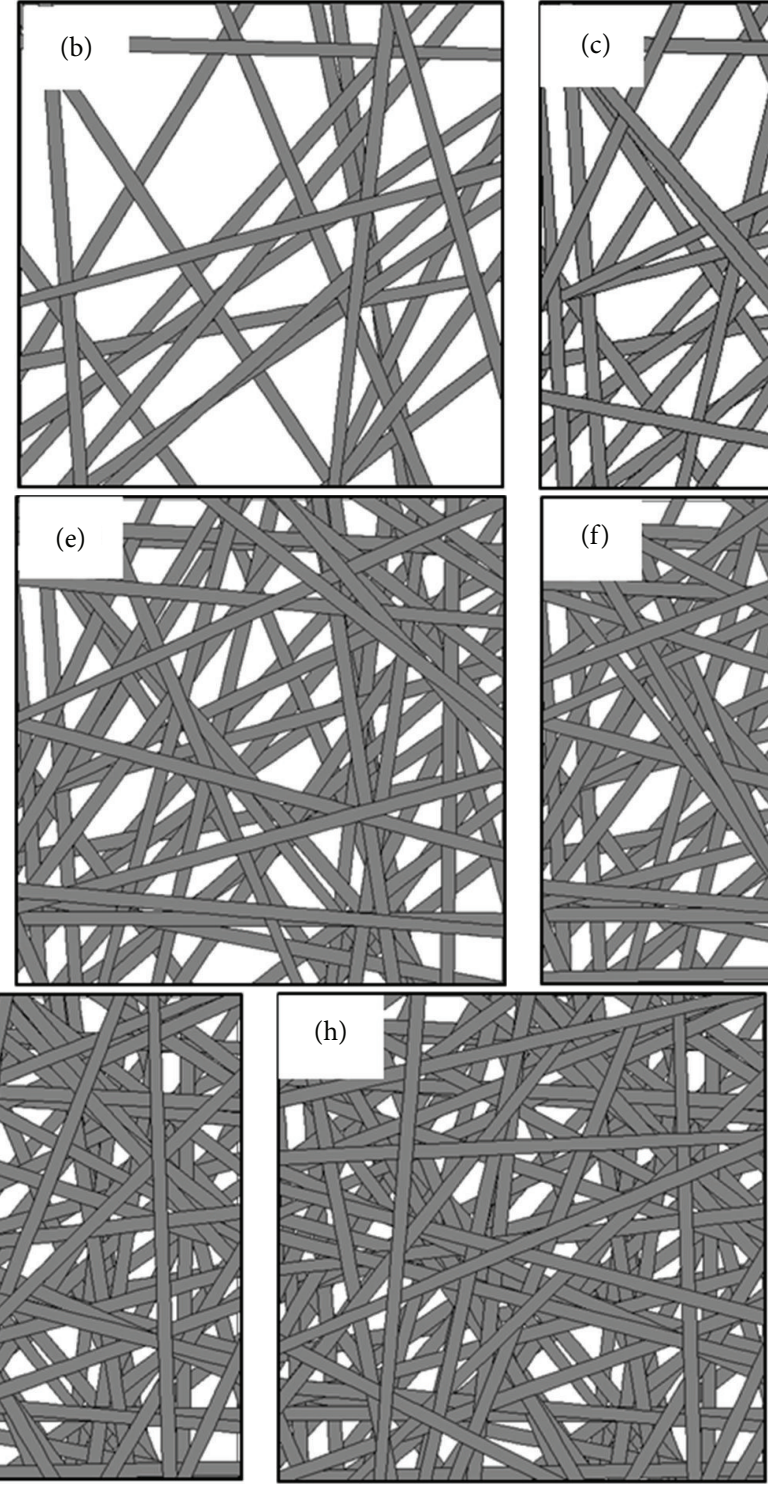

FIGURE 2: The simulated structures of nanofibers with monodisperse size of $d_{f}$ when the number density of fibers is (a) 0.1 , (b) 0.2 , (c) 0.3 , (d) 0.4 , (e) 0.5 , (f) 0.6 , (g) 0.7 , and (h) 0.8 .

done by the program. These monotonous results show that all samples had the same coverage area even though the fiber sizes could be differentiated. This happens probably because the chosen size distribution is still too close to each other and all distributions have the same average size which is $d_{f}$. This may be considered as a confirmation that if the average size of nanofibers could be controlled to be equal for all samples, the surface coverage of the fibers could be pretty much the same, independent on the fiber size distribution in the ranges studied here.

The effect of number density on the surface coverage is clearly seen in Figure 6. The coverage certainly increases upon increasing the number density but the increase rate is slower at higher density due to higher chance of fiber overlaps as seen in two dimensions. However, upon adding more fibers, the projected pore sizes of the structures decrease as shown in Figure 7. In the figure, it is obvious that an increase in fiber density could decrease the pore size of nanostructures rapidly at low fiber density. The pore size decrease rate is lower when the fiber density increases due to less probability for fibers to deposit on the vacant area. The transition is seen at density of 0.2 or it may be thought as a crossover of the pore generation mechanism. At a high fiber density on the surface, it could be seen that there are lots of small pores the size of which is around 1-2 times the fiber size. Figure 7 also shows similar results of the average pore areas when the size distribution was varied. The discussion was already given earlier. However, the difference was clearly seen at the low coverage of the fiber. It seems that for a low fiber density, the distribution that contains the larger size range results in smaller pore area. The $2 \mathrm{D}$ structure at a low density is important in simulation of nanofibers in 3 dimensions where the whole membrane is made of layers of thin 2D networks [11]. 

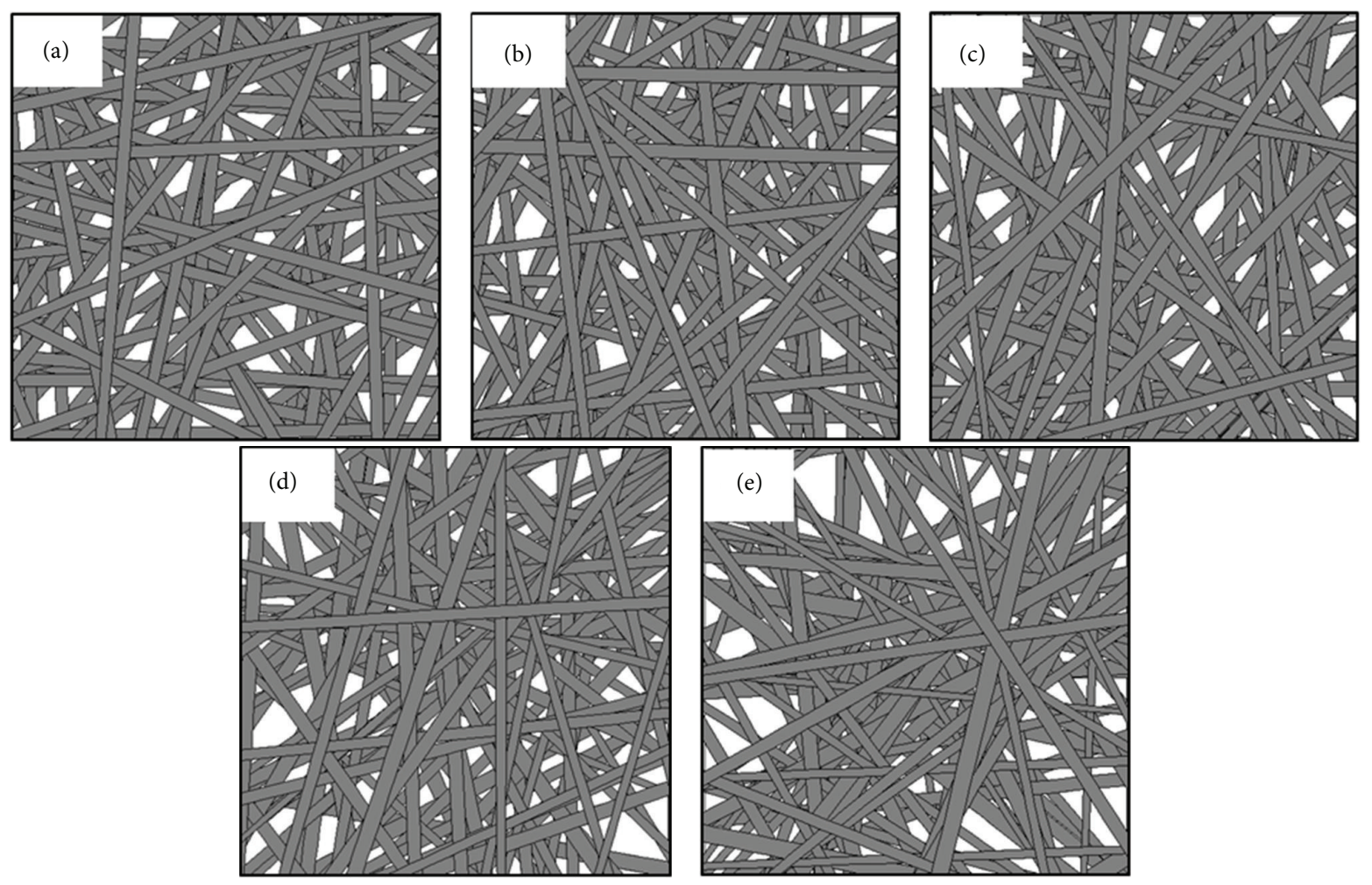

FIGURE 3: Nanofibrous structures with various size polydispersity: (a) monodisperse size of $d_{f}$, (b) $0.85 d_{f}-1.15 d_{f}$, (c) $0.75 d_{f}-1.25 d_{f}$, (d) $0.65 d_{f}-1.35 d_{f}$, and (e) $0.55 d_{f}-1.45 d_{f}$.
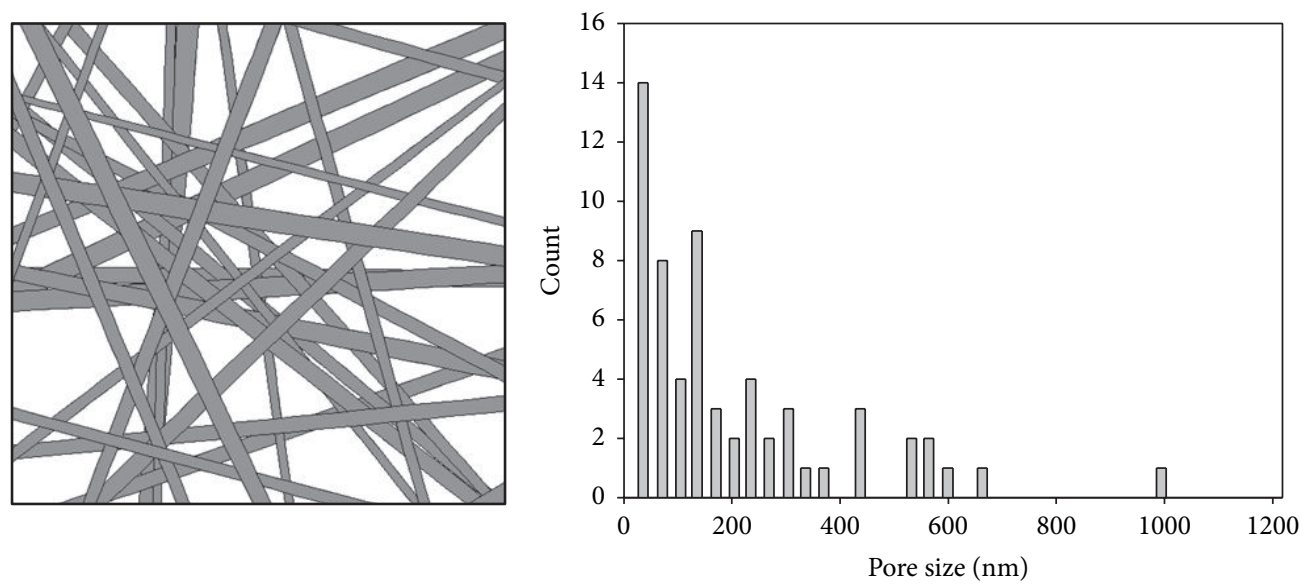

Figure 4: The simulated N6-35 nanofibrous structures and its pore size distribution to be compared with Figure 1.

3.5. The Probability of a Particle Passing through 2D Pores. Since one of the applications of nanofibers is being used as a filter, with these simulated structures, it is possible to obtain the probability for a particle to successfully move through the pores perpendicular to the direction of the flow. In this test, particles are sampled one by one to be deposited in the pore as described by ballistic deposition model. The number of attempts made to successfully place the particle in the pore, that is, the particle does not overlap with the fibers, is counted. Therefore, the reciprocal of this number is the probability of the particle passing through the filter without being intercepted by the fibers. The results are shown in Figure 8, where the size of the particles is varied while the size of the fibers is fixed at $d_{f}$.

It is found that at the same fiber number density, larger particles tend to stick more easily to the fibers than the smaller particles, yielding higher efficiency of the filtration [16]. However, in a real situation small particles could aggregate because of their high surface energy which results in large agglomerates leading to a high efficiency in filtration. It was reported that there is an optimum size range for particles to easily get through the filter [9]. Particles smaller 


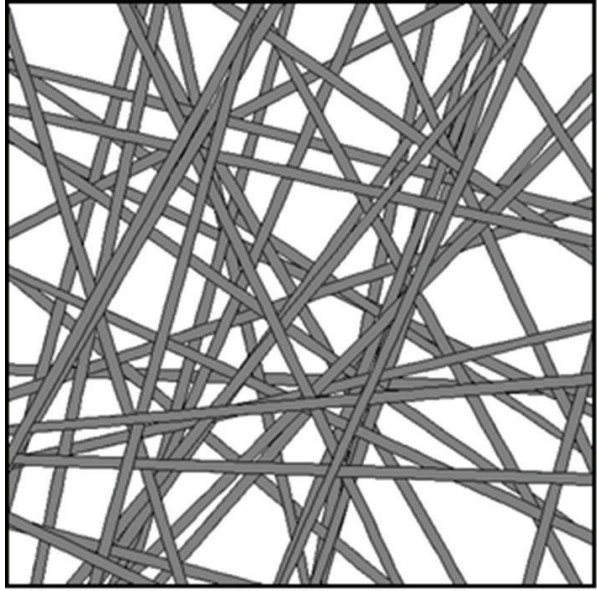

(a)

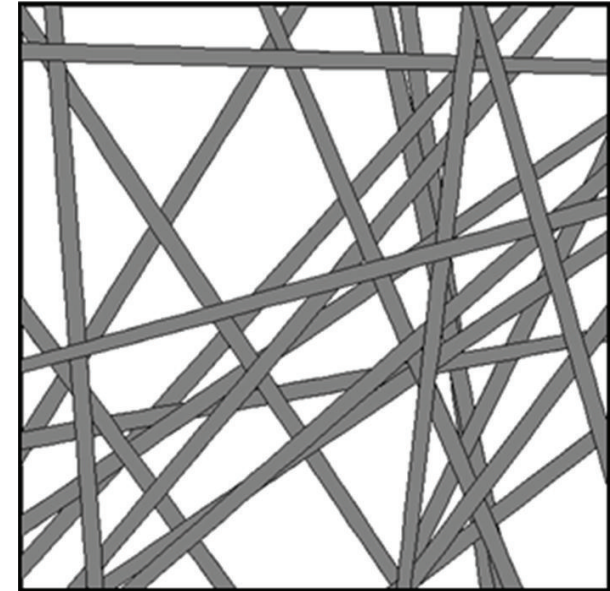

(b)

Figure 5: The comparison of simulated structures for N6-30 (size around $100 \mathrm{~nm}$ ) and N6-35 (size around $150 \mathrm{~nm}$ ) at the same solid volume fraction.

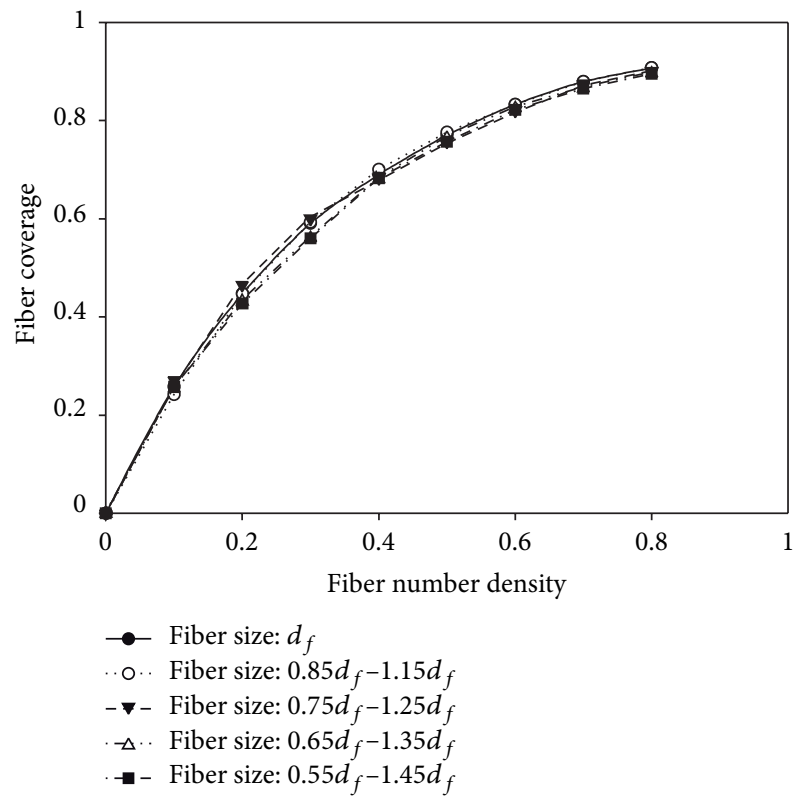

FIGURE 6: Surface coverage of nanofibers at various number density for various size distribution.

than this size range could form an aggregate which is larger than the pore size and particles larger than this size range are larger than the pore size. The topic of aggregation will be investigated more in the future. The number density in this case actually correlates with the thickness of the filter in 3D. As the number of fiber increases, the thickness of the membrane increases and this certainly increases the filtration efficiency with an expense of high pressure drop across the membrane [15].

The experimental study of filtration of small particles with nylon-6 nanofibers was also done in this work. With a membrane the thickness of which is around $0.1 \mathrm{~mm}$, the N6-30 samples could completely remove all particles from

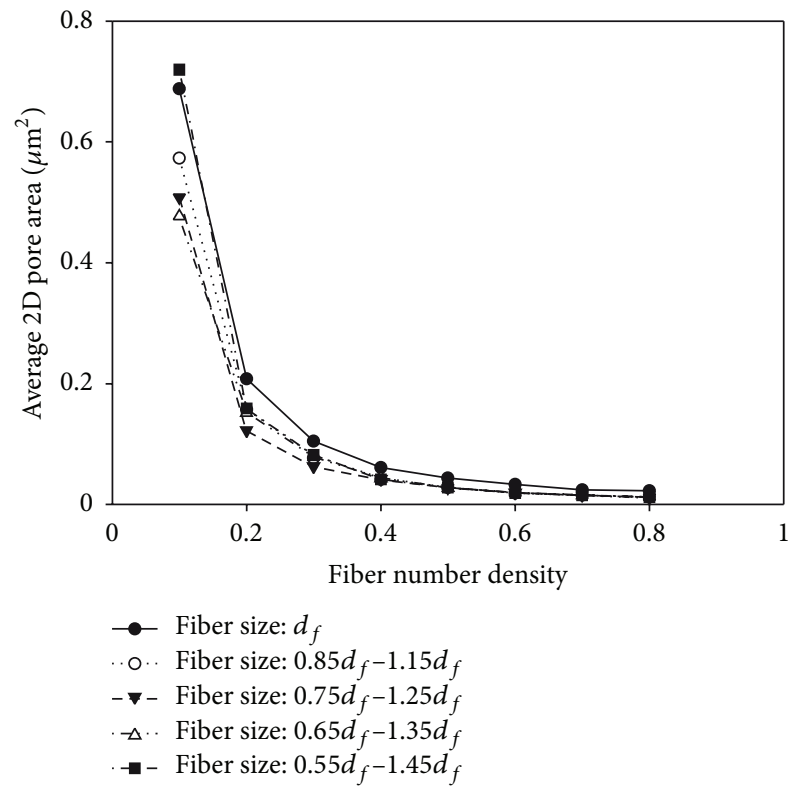

FIGURE 7: The average projected pore areas in squared micron (as seen in 2D) at different fiber number density. The comparison is for all distributions.

the 200 ppm polystyrene particle suspension; that is, the separation efficiency according to (2) equals $100 \%$. Figure 9 shows the filter fronts where particles with diameters of $90 \mathrm{~nm}$ and $200 \mathrm{~nm}$ were captured. The outline of the nanofibers are still seen for the case of smaller particles while almost complete surface coverage is seen for the larger ones, implying higher probability for smaller particles to move inside the pores of nanofibrous structures as was seen in the simulations.

\subsection{The Void Volume Fraction and Tortuosity of the Structures.} Based on the Kozeny-Carman relation, which could well describe the pressure drop of laminar flow through fibrous mats, the void volume fraction of the nanostructures could 


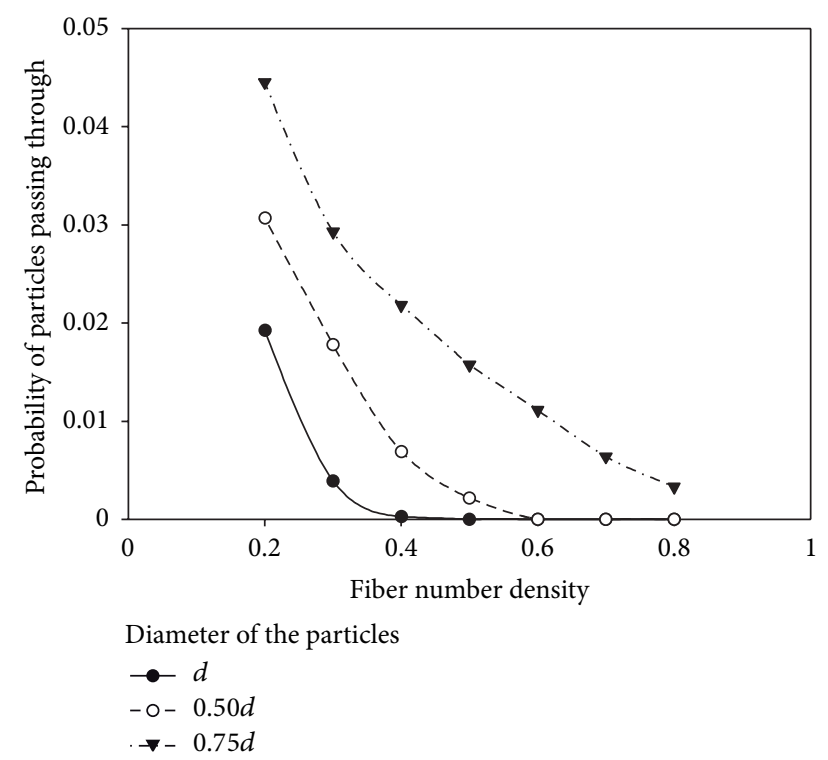

FIgURE 8: The probability for a particle with different size to successfully move through the $2 \mathrm{D}$ pore in the structures made of various fiber densities.

be estimated [17]. The equation was modified here to take into account the tortuosity of the structures and the actual velocity of the flow:

$$
\frac{\Delta P}{\tau z}=\frac{16 k \mu U_{o}}{d_{f}^{2}} \frac{(1-\varepsilon)^{2}}{\varepsilon^{4}}
$$

In (3), $U_{o}$ is the superficial velocity $(\mathrm{m} / \mathrm{s}), \mu$ is the viscosity of water at $30^{\circ} \mathrm{C}(\mathrm{Pa} \cdot \mathrm{s}), z$ is the filter thickness $(\mathrm{m}), \Delta P$ is pressure drop $(\mathrm{Pa}), \varepsilon$ is the void fraction, and $\tau$ is the tortuosity; $k$ is the Kozeny constant, which is equal to 0.55 for nearly cylindrical fibers if void fraction $(\varepsilon)$ varies from 0.6 to 0.8 .

The tortuosity could be approximated by realization that the whole fibrous mat is composed of many layers of nanofiber networks. Each layer is very thin and the surface coverage is equal to $\theta$. Thus, the probability of liquid to pass through each layer is $1-\theta$, which is equivalent to void fraction, $\varepsilon$. The thin layer of thickness $d z$ is considered and the liquid is divided into $N$ parts. If some parts of the liquid could pass directly through this layer then the traveling distance of these parts is $\varepsilon N d z$. The other parts are blocked by the fibers so the liquid has to move laterally for a distance $2 N(1-\varepsilon) \varepsilon d z$, in case the available pore is located at the distance $d z$ from the fiber. Still there are liquid parts that are blocked by the fibers and have to travel farther with a distance $3 N(1-\varepsilon)^{2} \varepsilon d z$. It is obvious that the probability for the liquid to find the pore depends on the void volume fraction. With this logic, one could write the equation of tortuosity which is defined as the ratio of actual distance for the liquid to travel to
TABLE 1: The void volume fraction of the nylon- 6 nanofibrous structures.

\begin{tabular}{lcccc}
\hline Sample & Thickness $(\mathrm{m})$ & Time $(\mathrm{s})$ & Void fraction & Tortuosity \\
\hline N6-30 & $1.04 E-04$ & 112.34 & 0.748 & 1.34 \\
N6-35 & $1.16 E-04$ & 93.66 & 0.719 & 1.39 \\
\hline
\end{tabular}

TABLE 2: The pore sizes from Hagen-Poiseuille equation and from SEM images.

\begin{tabular}{cccc}
\hline Sample & $\begin{array}{c}\text { Pore size from } \\
\text { Hagen-Poiseuille }(\mathrm{nm})\end{array}$ & $\begin{array}{c}\text { Pore size from } \\
\text { SEM }(\mathrm{nm})\end{array}$ & $\begin{array}{c}\text { Fiber diameter } \\
(\mathrm{nm})\end{array}$ \\
\hline N6-30 & 181 & 94 & 103 \\
N6-35 & 216 & 154 & 144 \\
\hline
\end{tabular}

the mat thickness. The actual distance is the average of traveling distance of all liquid parts. Therefore, we have

$$
\begin{aligned}
\tau & =\frac{D_{\text {travel,ave }}}{d z} \\
& =\frac{\varepsilon N+2 N(1-\varepsilon) \varepsilon+3 N(1-\varepsilon)^{2} \varepsilon+4 N(1-\varepsilon)^{3} \varepsilon+\cdots}{N} .
\end{aligned}
$$

The number of terms in (4) could reach infinity so tortuosity could be approximated as

$$
\tau=\frac{1}{\varepsilon}
$$

The fixed volume of water was fixed at $4.60 \times 10^{-6} \mathrm{~m}^{3}$ and the weight pushing the water through the filter was also fixed at $6.42 \mathrm{~N}$. The filtration time was recorded as shown in Table 1 and the void fractions of N6-30 and N6-35 mats can be calculated.

It is noted that as seen from the SEM images in Figure 1 and simulated images in Figure 5, the pore size is larger when the deposited fibers are larger and this could affect the void fraction as a whole so N6-35 had higher void fraction than N6-30. Based on this value, one could assume the first layer is the network with surface coverage equal to $1-\varepsilon$, which is 0.252 and 0.281 for N6-30 and N6-35, respectively. According to Figure 6, for N6-35 with the surface coverage about 0.3, the number density is a bit larger than 0.1. If the structure with density of 0.1 is adopted for the first layer, the multiple layers could be built layer by layer to obtain the whole mat as previously done by Sambaer et al. [11]. Thus, using the connection of the experimental results and simulated results, one could have a guide to construct the structure of each layer.

3.7. The Average Pore Size Determination. Based on the water flux measurement as discussed in the previous section, the Hagen-Poiseuille equation, (1), could be used to estimate the volume-averaged pore size [18]. The equation was derived for the flow in circular pipe so, in this work, the channel inside the structure is assumed to be little tortuous circular pipes with nonuniform cross-sectional area. The volume-averaged pore size was obtained as shown in Table 2. 


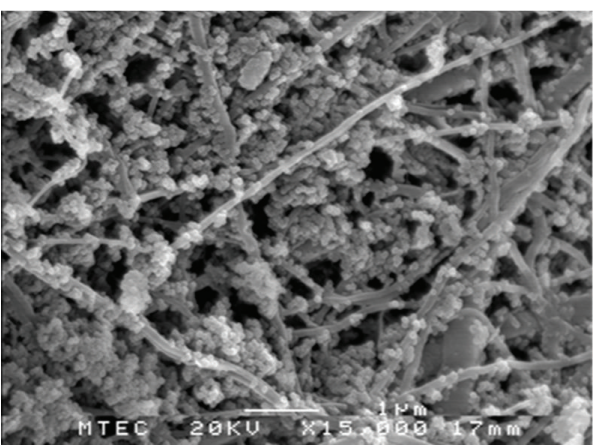

(a)

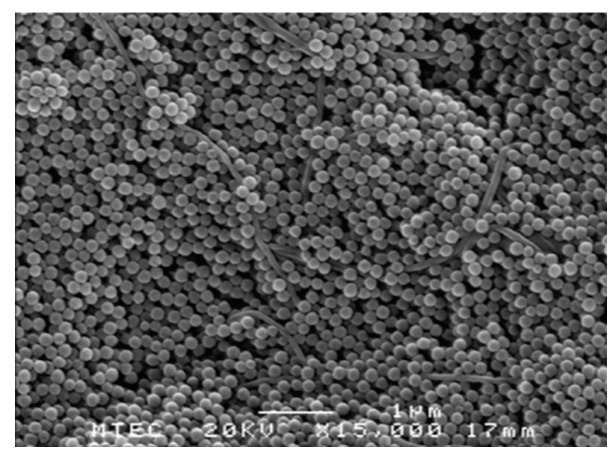

(b)

FIGURE 9: SEM images of polystyrene particles with different diameters, (a) $90 \mathrm{~nm}$ and (b) $200 \mathrm{~nm}$, on the filter fronts.

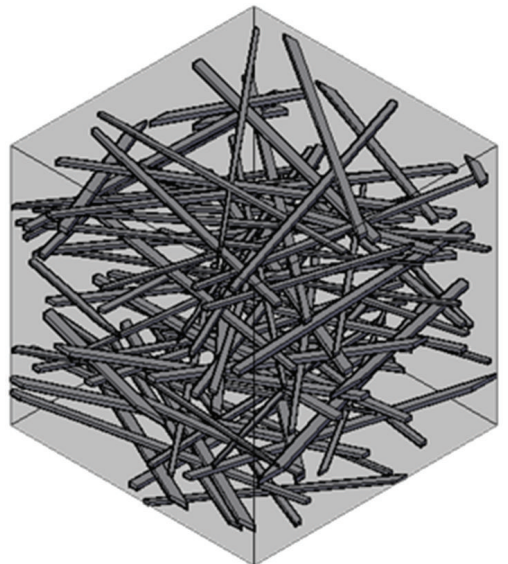

(a)

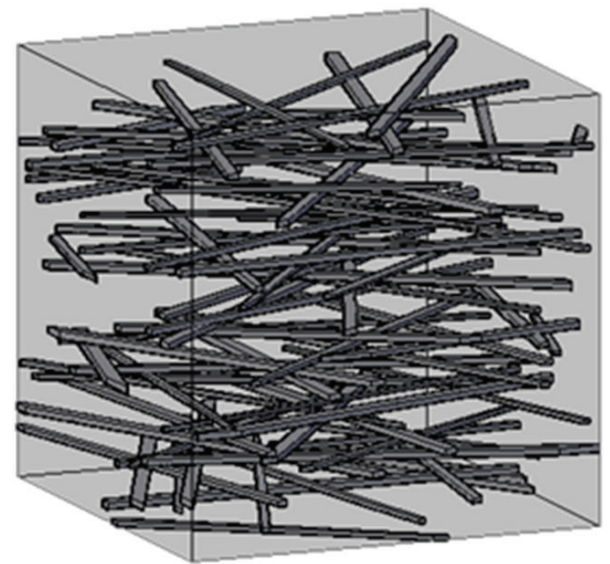

(b)

FIgURE 10: An example of 3D structures of nanofibers.

From Table 2, it is found that the volume-averaged pore size obtained from Hagen-Poiseille equation is larger than the pore size obtained from SEM figures. If the volume-averaged pore size is considered as $3 \mathrm{D}$ pore size, $d_{3 \mathrm{D}}$, and the average from SEM image as $2 \mathrm{D}$ pore size, $d_{2 \mathrm{D}}$, it is possible to relate two quantities by

$$
d_{2 \mathrm{D}}^{2}+d_{f}^{2}=d_{3 \mathrm{D}}^{2}
$$

Equation (6) was used to estimate $d_{3 \mathrm{D}}$ for N6-30 and N6-35 and it is found that $d_{3 \mathrm{D}}$ 's are $139 \mathrm{~nm}$ and $210 \mathrm{~nm}$, respectively, being compared with 181 and $216 \mathrm{~nm}$. The numbers imply that in three dimensions, the pores are generated by the deposition of one fiber on top of other fibers so the space along the axis of membrane thickness between two fibers is at least equal to the fiber diameter as was assumed when $3 \mathrm{D}$ simulated structures were created by Hosseini and Tafreshi [10]. This is seen in our example of 3D structure of N6-35 shown in Figure 10. The structures were built by adding one fiber at a time on top of the previously added fiber.

\section{Conclusions}

The simulation of nanofibrous structures via Monte Carlo method in 2 dimensions was performed. The results confirmed that even though there are some variations in fiber size, if the average size of fiber could be controlled, the surface coverage of fibers randomly deposited during electrospinning process could be the same. However, at a low number density of fiber, the average pore size was different when the fiber size range was varied. This structure may be considered as a structure of initial layers that could be used to simulate the $3 \mathrm{D}$ structures by combining multiple thin 2D networks together as a whole mat.

The simulated structures resembled real SEM images of nanofibers closely since the fiber size distribution and the average size of the fibers were chosen to be close to the real systems, confirming efficiency of $2 \mathrm{D}$ simulations. Calculations of void fraction and tortuosity of the structures could be used to prove that the space between two fibers along the axis of membrane thickness is at least equal to the size of the fibers deposited, providing the connection between $3 \mathrm{D}$ pore size and 2D pore size from the SEM figures or from the simulated 2D figures.

\section{Conflict of Interests}

The authors do not have any conflict of interests regarding this research work. 


\section{Acknowledgments}

This research was jointly funded by the Thailand Research Fund (TRF) and Thammasat University (Grant no. DBG5380007). All assistance from the Faculty of Engineering is highly appreciated.

\section{References}

[1] S.-W. Choi, A. Katoch, G. -J. Sun, and S. S. Kim, "Synthesis and gas sensing performance of $\mathrm{ZnO}-\mathrm{SnO}_{2}$ nanofiber-nanowire stem-branch heterostructure," Sensors and Actuators B, vol. 181, pp. 787-794, 2013.

[2] H. R. Pant, D. R. Pandeya, K. T. Nam, W.-I. Baek, S. T. Hong, and H. Y. Kim, "Photocatalytic and antibacterial properties of a $\mathrm{TiO}_{2} /$ nylon-6 electrospun nanocomposite mat containing silver nanoparticles," Journal of Hazardous Materials, vol. 189, no. 1-2, pp. 465-471, 2011.

[3] D.-G. Yu, W. Chian, X. Wang, X.-Y. Li, Y. Li, and Y.-Z. Liao, "Linear drug release membrane prepared by a modified coaxial electrospinning process," Journal of Membrane Science, vol. 428, pp. 150-156, 2013.

[4] R. Nirmala, R. Navamathavan, H.-S. Kang, M. H. El-Newehy, and H. Y. Kim, "Preparation of polyamide-6/chitosan composite nanofibers by a single solvent system via electrospinning for biomedical applications," Colloids and Surfaces B, vol. 83, no. 1, pp. 173-178, 2011.

[5] K. Desai, K. Kit, J. Li, P. Michael Davidson, S. Zivanovic, and H. Meyer, "Nanofibrous chitosan non-wovens for filtration applications," Polymer, vol. 50, no. 15, pp. 3661-3669, 2009.

[6] P. Danwanichakul, D. Dechojarassri, and R. Werathirachot, "An approximate model describing electrospinning of nanofibers: process parameter investigation," International Journal of Electrospun Nanofibers and Applications, vol. 2, pp. 103-114, 2008.

[7] H.-S. Park and Y. O. Park, "Filtration properties of electrospun ultrafine fiber webs," Korean Journal of Chemical Engineering, vol. 22, no. 1, pp. 165-172, 2005.

[8] S. J. Eichhorn and W. W. Sampson, "Statistical geometry of pores and statistics of porous nanofibrous assemblies," Journal of the Royal Society Interface, vol. 2, no. 4, pp. 309-318, 2005.

[9] B. Maze, H. Vahedi Tafreshi, Q. Wang, and B. Pourdeyhimi, "A simulation of unsteady-state filtration via nanofiber media at reduced operating pressures," Journal of Aerosol Science, vol. 38, no. 5, pp. 550-571, 2007.

[10] S. A. Hosseini and H. V. Tafreshi, "3-D simulation of particle filtration in electrospun nanofibrous filters," Powder Technology, vol. 201, no. 2, pp. 153-160, 2010.

[11] W. Sambaer, M. Zatloukal, and D. Kimmer, "3D modeling of filtration process via polyurethane nanofiber based nonwoven filters prepared by electrospinning process," Chemical Engineering Science, vol. 66, no. 4, pp. 613-623, 2011.

[12] S. Zhang, W. S. Shim, and J. Kim, "Design of ultra-fine nonwovens via electrospinning of Nylon 6: spinning parameters and filtration efficiency," Materials and Design, vol. 30, no. 9, pp. 3659-3666, 2009.

[13] D. Hussain, F. Loyal, A. Greiner, and J. H. Wendorff, "Structure property correlations for electrospun nanofiber nonwovens," Polymer, vol. 51, no. 17, pp. 3989-3997, 2010.

[14] A. Thorvaldsson, P. Edvinsson, A. Glantz, K. Rodriguez, P. Walkenstrim, and P. Gatenholm, "Superhydrophobic behaviour of plasma modified electrospun cellulose nanofiber-coated microfibers," Cellulose, vol. 19, pp. 1743-1748, 2012.
[15] J. Wang, S. C. Kim, and D. Y. H. Pui, "Investigation of the figure of merit for filters with a single nanofiber layer on a substrate," Journal of Aerosol Science, vol. 39, no. 4, pp. 323-334, 2008.

[16] D. Aussawasathien, C. Teerawattananon, and A. Vongachariya, "Separation of micron to sub-micron particles from water: electrospun nylon-6 nanofibrous membranes as pre-filters," Journal of Membrane Science, vol. 315, no. 1-2, pp. 11-19, 2008.

[17] E. Mauret and M. Renaud, "Transport phenomena in multiparticle systems-I. Limits of applicability of capillary model in high voidage beds-application to fixed beds of fibers and fluidized beds of spheres," Chemical Engineering Science, vol. 52, no. 11, pp. 1807-1817, 1997.

[18] P. Johnson, Fluid Sterilization by Filtration, Interpharm/CRC, New York, NY, USA, 3rd edition, 2003. 

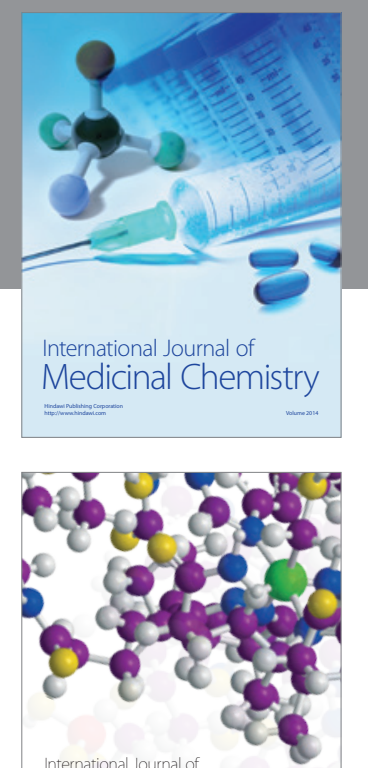

\section{Carbohydrate} Chemistry

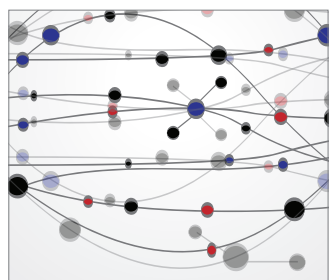

The Scientific World Journal
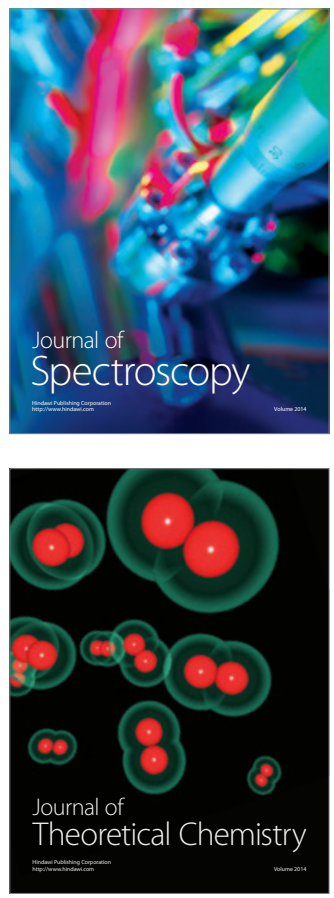
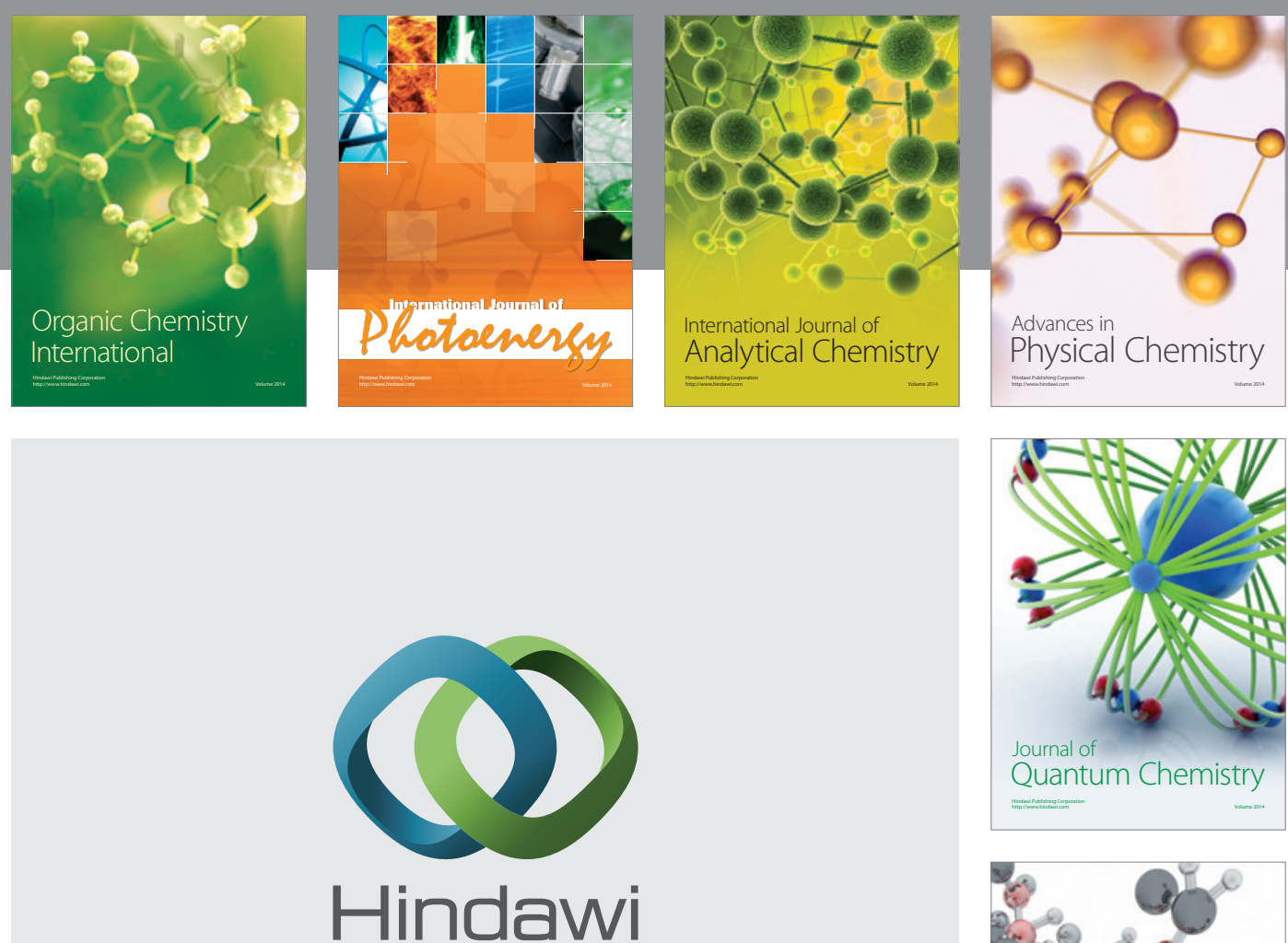

Submit your manuscripts at

http://www.hindawi.com

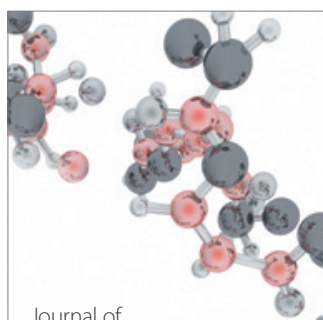

Analytical Methods

in Chemistry

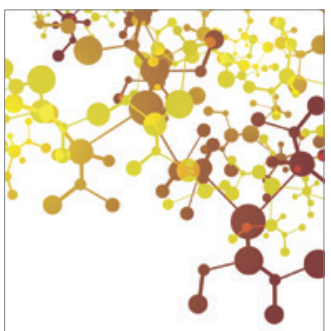

Journal of

Applied Chemistry

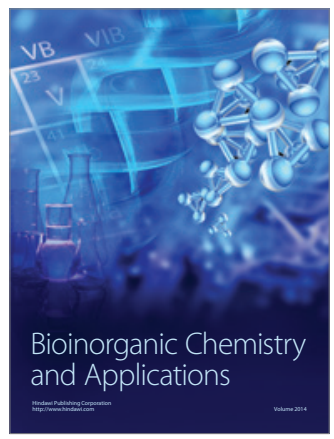

Inorganic Chemistry
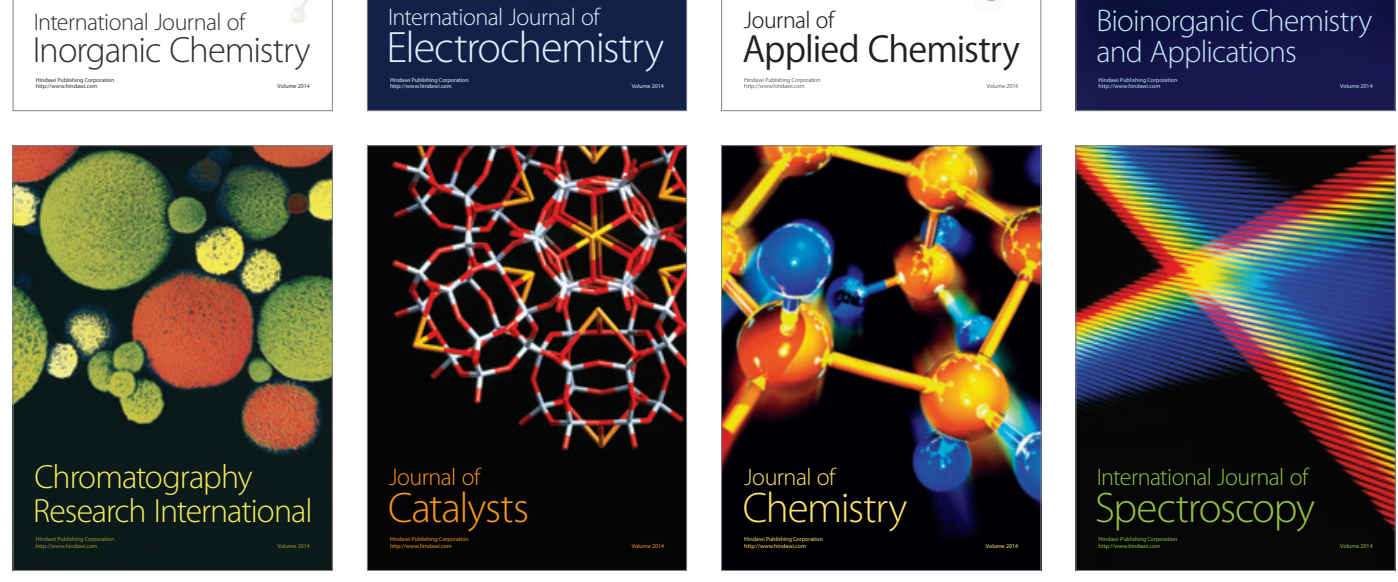